\title{
Predictive Value of Circulatingve-catherin in Coronary Artery Disease Patients with Symptomatic Moderate to Severe Chronic Heart Failure
}

ALEXANDER E. BEREZIN, ${ }^{1}$ ALEXANDER A. KREMZER, ${ }^{2}$ TATAYNA A. SAMURA ${ }^{2}$

\begin{abstract}
Aim: To evaluate the prognostic value of circulating VE-catherinfor cumulative survivalin patients with ischemic chronic heart failure (CHF).

Methods: A total of I54 patients withischemic symptomatic moderate-to-severe CHF were enrolled in the study on discharge from the hospital. Observation period was up to 3 years. Blood samples for biomarkers measurements were collected. ELISA method for measurements of circulating level of VE-catherin was used. Concentrations of VEcatherinfor cumulative survival cases due to advanced CHF were tested. Additionally, all-cause mortality, and CHFrelated death were examined.

Results: During a median follow-up of 2.18 years, 21 participants died and 106 subjects were hospitalized repetitively. Medians of circulating levels of VE-catherin in survived and died patient cohort were $0.63 \mathrm{ng} / \mathrm{ml}(95 \%$ confidence interval $[\mathrm{Cl}]=0.55-0.64 \mathrm{ng} / \mathrm{ml})$ and $1.03 \mathrm{ng} / \mathrm{ml}(95 \% \mathrm{Cl}=0.97-1.07 \mathrm{ng} / \mathrm{ml})(\mathrm{P}<0.00 \mathrm{I})$. ROC analysis has been shown that cutoff point of VE-catherin concentration for cumulative survival function was $0.755 \mathrm{ng} / \mathrm{ml}$. It has beenfound a significantly divergence of Kaplan-Meier survival curves in patients with high (>0.755 ng/ml) and low $(<0.755 \mathrm{ng} / \mathrm{ml})$ concentrations of VE-catherin. Circulating VE-catherin independently predicted all-cause mortality $(O R=1.27 ; 95 \% C l=1.08-1.59 ; P=0.002)$, CHF-related death $(O R=1.16 ; 95 \% \mathrm{Cl} I .02-1.50 ; P<0.001)$, and also CHF-related rehospitalisation $(O R=I .12 ; 95 \% \mathrm{Cl}=1.07-1.25 ; P<0.00 I)$ within 3 years of observation period.
\end{abstract}

Conclusion: Increased circulating VE-catherinassociates with increased 3-year CHF-related death, all-cause mortality, and risk for recurrent hospitalization due to CHF.

Key words: VE-catherin; chronic heart failure; survival; hospitalization; prognosis.

\section{Introduction}

Chronic heart failure (CHF) is considered as a leading cause of morbidity and mortality inworldwide. ${ }^{1}$ Nature evolution of CHF is associated with endothelial dysfunction that results in shear stress disorders on endothelium, which is exerted several factors, such as angiotensin-aldosterone system activation, oxidative stress, proinflammatory response, exaggerated collagen synthesisand extracellular matrix remodelling in vasculature, degradation of vasodilators etc. ${ }^{2}$ However, mechanical interaction of endothelial cells (ECs), which is mediated by blood flow,might lead to junction vascular remodelling. ${ }^{3}$ Particularly this phenomenon is

1. State Medical University, Internal Medicine Department, Zaporozhye, Ukraine

2. State Medical University, Clinical Pharmacology Department, Zaporozhye, Ukraine

Correspondence: Dr. Alexander E. Berezin, Professor, MD, PhD, Chief of Cardiology unit, Internal medicine Department, State Medical University, 26, Mayakovsky av., Zaporozhye, Postcode 69035, Ukraine. Tel.: +38 0612729607 Fax: +38 061 2729607, E-mail: dr_berezin@mail.ru realized by involvement of junctional protein vascular endothelial-cadherin (VE-cadherin) that is essential for the endothelial survival function. ${ }^{4}$ Currently it has known that VE-cadherin is a component of endothelial cell-to-cell adherens junctions, and it has a key role in the maintenance of vascular integrity. ${ }^{4}$ The mechanisms of action of VEcadherin are complex and include reshaping and organization of the ECs cytoskeleton and modulation of gene transcription. ${ }^{5}$ Therefore, VE-cadherin mediates cell adhesion and monolayer integrity, regulates angiogenesis and actin-driven to be support a mechanical capacity of endothelium after activation by soluble vascular cell adhesion molecules. ${ }^{6}$ There is currently evidence affected VE-cadherin as a marker of endothelial dysfunction in several settings. ${ }^{7}$ However, the prognostic value of circulating VE-catherin in the ischemic chronic heart failure has not been defined.

The objective of this study was toevaluate the prognostic value of circulating VE-catherin for cumulative survival in patients with ischemic chronic heart failure. 


\section{Methods}

The study evolved 154 patients (86 males) aged 48 to 62 years withischemic symptomatic moderate-to-severe CHF. Chronic heart failure (CHF) was diagnosed according to current clinical guidelines. ${ }^{8}$ Table 1 shows characteristic of the patients participated in the study. All the patients have given their written informed consent for participation in the study. The following are exclusion criteria: Q-wave and nonQ-wave MI within 3 months before study entry; severe kidney and liver diseases that may affect clinical outcomes; malignancy; creatinin plasma level above 440 ìmol/L; estimated GFR index $<35 \mathrm{ml} / \mathrm{min} / \mathrm{i}^{2}$; brain injury within 3 months before the enrollment; body mass index above 30 $\mathrm{kg} / \mathrm{m}^{2}$ and less $15 \mathrm{~kg} / \mathrm{m}^{2}$; pulmonary edema; tachyarrhythmia; valvular heart disease; thyrotoxicosis; ischemic stroke; intracranial hemorrhage; acute infections; surgery; trauma; all the ischemic events within 3 previous months; inflammations within a previous month; neoplasm; pregnancy; implanted pacemaker, any disorder that may discontinue patient's participation in the study according to investigators; and patient's refusal to participate in the study or to give his consent for it. Observation period was up to 3 years. We analyzed cumulative survival related to CHF, and additionally all-cause mortality was examined.

\section{Methods for visualization of coronary arteries}

Multispiral computed tomography angiography and/or angiographic study have been carried out to verify the ischemic nature of the disease in patients. Multispiral computed tomography angiography has been carried out for all the patients prior to their inclusion in the study. When atherosclerotic lesions of the coronary arteries were verified, patients were subjected to conventional angiographic examination provided indications for revascularization were available. CAD was considered to be diagnosed upon availability of previous angiographic examinations carried out not later than 6 months ago provided no new cardiovascular events occurred for this period, and the procedure are available for assay. The coronary artery wall structure was measured by means of contrast spiral computed tomography angiography ${ }^{9}$ on SomatomVolum Zoom scanner (Siemens, Erlangen, Germany) with two detector rows when holding patientsbreathe at the end of breathing in. After preliminary native scanning, non-ionic contrast Omnipak (Amersham Health, Ireland) was administered for the optimal image of the coronary arteries. To reconstruct the image, 0.6-mm-width axial tomographic slices were used.

\section{Assessment of hemodynamics}

Transthoracic ultrasonic echocardiography was performed according to a conventional procedure on ACUSON apparatus, SIEMENS, Germany, in Â-mode regimen and tissue Doppler echocardiography regimen from parasternal, subcostal, and apical positions over the short and long axis with sensor p of $5 \mathrm{mHz}$. Left ventricular end-diastolic and end-systolic volumes were measured by modified Simpson's planimetric method; and they were measured by cylinder method if severe failure of local myocardial contractility was verified. Left ventricular ejection fraction (LVEF) was assessed in compliance with the requirements of American Society of Echocardiography. ${ }^{10}$ Tissue Doppler echocardiography was carried out in 4-, 3- and 2-chamber projections in each of 16 segments of the left ventricle and in 4 spots of the mitral annulus: at the base of posterior septal, lateral, inferior, and anterior left ventricular walls. ${ }^{11}$ Peak systolic (Sm), early diastolic (Em), and late diastolic (Àm) myocardial velocities were measured in the mitral annulus area, followed by calculating velocity of early diastolic left ventricular filling (E) to Am (A/Àm) ratio and to Em (A/ Em) ratio.

\section{Calculation of glomerular filtration rate}

Calculation of glomerular filtration rate (GFR) was carried out using MDRD-6 formula. ${ }^{11}$

\section{Measurement of VE-catherin, NT-pro-BNP, total cholesterol and its fractions}

To determine VE-catherin, N-terminal pro-brain natriuretic peptide (NT-pro-BNP), total cholesterol and cholesterol fractions, blood samples were drawn in the morning (at 7-8 a.m.) into cooled silicone test tubes. Samples were processed according to the recommendations of the manufacturer of the analytical technique used.They were centrifuged upon permanent cooling at 6,000 rpm for 3 minutes. Then, plasma was refrigerated immediately to be stored at a temperature not higher than $-35^{\circ} \mathrm{C}$. Circulating VE-catherin level was determined by ELISA method (Bender MedSystems GmbH, Vienna, Austria). NT-pro-BNP content was measured by immunoelectrochemoluminescent assay using sets by R\&D Systems (USA) on Elecsys 1010 analyzer (Roche, Mannheim, Germany). Concentrations of total cholesterol (TC) and cholesterol of high-density lipoproteins (HDLP) were measured by fermentation method. Concentration of cholesterol of low-density lipoproteins (LDL-C) was calculated according to the Friedewald formula (1972). ${ }^{12}$

\section{Statistical Analysis}

Statistical analysis of the results obtained was carried out in SPSS system for Windows, Version 22 (SPSS Inc, Chicago, IL, USA). The data were presented as mean (M) and error of mean ( $\pm \mathrm{m}$ ) or $95 \%$ confidence interval (CI); median (Me) and interquartile range. The hypothesis of normal distribution of the parameters analyzed was checked by means of Shapiro-Wilk test and Kolmogorov-Smirnovtest. To compare the main parameters of patients' groups (subject to 
the type of distribution of the parameters analyzed), onetailed Student $t$-test or Shapiro-Wilk U-test were used. To compare categorical variablesbetween groups, $\mathrm{Chi}^{2}$ test $\left(\chi^{2}\right)$ and Fisher $\mathrm{F}$ exact test were used. The circulating VEcatherin and NT-pro-BNP level in the blood failed to have a normal distribution, while distribution of the total cholesterol and cholesterol fractions had a normal character (estimated by means of Kolmogorov-Smirnovtest) and was not subjected to any mathematical transformation. The factors, which could be associated potentially with circulating VE- catherin, were determined by logistic regression analysis. Receive Operation Curve (ROC) analysis was carried out to identify the optimal cutoff points of the VE-catherin concentration with predicted value. Odds ratio (OR) and 95\% confidence interval (95\% CI) were calculated for all the independent predictorsof survival of the patients. A calculated difference of $\mathrm{P}<0.05$ was considered significant.

\section{Results}

General characteristics of study patient population

Table-I

General characteristic of patients participating in the study

\begin{tabular}{|c|c|c|}
\hline Variable & Died subjects $(n=21)$ & Survivedsubjects $(\mathrm{n}=133)$ \\
\hline Age, years & $57.20 \pm 6.70$ & $59.50 \pm 7.30$ \\
\hline Males, n (\%) & $12(57.1 \%)$ & $67(50.3 \%)$ \\
\hline Arterial hypertension, n (\%) & $12(57.1 \%)$ & $61(45.9 \%)$ \\
\hline Hyperlipidemia, n (\%) & $9(42.8 \%)$ & $52(39.1 \%)$ \\
\hline T2DM, n (\%) & $8(38.1 \%)$ & $45(33.8 \%)$ \\
\hline Adherence to smoking, n (\%) & $7(33.3 \%)$ & $24(29.3 \%)$ \\
\hline \multicolumn{3}{|l|}{ NYHA Class } \\
\hline - $\quad$ II Class & $6(28.6 \%)$ & $35(26.3 \%)$ \\
\hline - $\quad$ III Class & $9(42.8 \%)$ & $65(48.9 \%)$ \\
\hline - IV Class & $6(28.6 \%)$ & $33(24.8 \%)$ \\
\hline BMI, $\mathrm{kg} / \mathrm{m}^{2}$ & 23.7 (95\% CI=22.5-27.3) & $24.2(95 \%$ CI=22.0-27.9) \\
\hline GFR, mL/min/1.73 m² & $82.1(95 \%$ CI=69.9-93.1) & 85.2 (95\% CI=70.3-112.5) \\
\hline HbA1c, \% & $6.3(95 \% \mathrm{CI}=4.4-9.0)$ & 7.0 (95\% CI=4.3-9.2) \\
\hline Fasting blood glucose, mmol/L & $4.80(95 \% \mathrm{CI}=3.6-8.5)$ & $5.40(95 \% \mathrm{CI}=3.4-9.1)$ \\
\hline Creatinine, ìmol/L & 70.5 (95\% CI=59.6-88.3) & $74.9(95 \%$ CI=65.1-90.3) \\
\hline Total cholesterol, mmol/L & $5.3(95 \% \mathrm{CI}=4.6-6.0)$ & $5.0(95 \% \mathrm{CI}=4.2-5.8)$ \\
\hline LDL-C, mmol/L & $3.60(95 \%$ CI $=3.20-4.18)$ & 3.02 (95\% CI=2.80-3.90) \\
\hline HDL-C, mmol/L & 0.94 (95\% CI = 0.92-1.06) & $0.88(95 \%$ CI = 0.82-0.97) \\
\hline NT-pro-BNP, pg /mL & 1533.6 (95\% CI644.5 - 2560.6) & $1031.2(95 \%$ CI $704.8-1560.7) *$ \\
\hline Systolic BP, mm Hg & $129 \pm 4$ & $135 \pm 5$ \\
\hline Heart rate, beats per $1 \mathrm{~min}$. & $76 \pm 6$ & $68 \pm 3$ \\
\hline LVEF, \% & $42.80 \pm 0.76$ & $55.40 \pm 0,80 *$ \\
\hline Å/Àm, U & $16.6 \pm 0.94$ & $16.5 \pm 1.20$ \\
\hline$\AA / \mathrm{Em}, \mathrm{U}$ & $16.6 \pm 1.00$ & $16.6 \pm 0.84$ \\
\hline \multicolumn{3}{|l|}{ Number of coronary arteries affected } \\
\hline • $\quad$ One-vessel lesion, n (\%) & $5(23.8 \%)$ & $24(18.0 \%)$ \\
\hline • $\quad$ Two-vessel lesion, n (\%) & $8(38.1 \%)$ & $54(40.1 \%)$ \\
\hline - $\quad$ Three- and multi-vessel lesion, n (\%) & $8(38.1 \%)$ & $55(41.4 \%)$ \\
\hline ACEI / ARAs, n (\%) & $21(100 \%)$ & $133(100 \%)$ \\
\hline acetylsalicylic acid, n (\%) & $19(90.5 \%)$ & $121(91.0 \%)$ \\
\hline Other antiaggregants, n (\%) & $2(9.5 \%)$ & $12(9.0 \%)$ \\
\hline Statins, n (\%) & $14(66.7 \%)$ & $80(60.2 \%)$ \\
\hline metformin, n (\%) & $8(38.1 \%)$ & $45(33.8 \%)$ \\
\hline diuretics, n (\%) & $18(85.7 \%)$ & $121(91.0 \%)$ \\
\hline Mineralcorticoid receptors antagonists, n (\%) & $9(42.9 \%)$ & $70(52.6 \%)$ \\
\hline
\end{tabular}

Note: * - statistically differences between parameters in the two groups $(\mathrm{P}<0.05)$; CI - confidence interval; CAD - coronary artery disease, T2DM - type two diabetes mellitus,GFR - Glomerular filtration rate, HDL-C - high-density lipoprotein cholesterol, LDL-C - Low-density lipoprotein cholesterol, BP - blood pressure, BMI - Body mass index, NYHA - New York Heart Association, BNP - brain natriuretic peptide, LVEF - Left ventricular ejection fraction, U - unit, Em - early diastolic myocardial velocity, Àm - late diastolic myocardial velocity, E - peak velocity of early diastolic left ventricular filling, ACEI - angiotensin-converting enzyme inhibitor, ARAs - angiotensin-2 receptors antagonists. 
During a median follow-up of 2.18 years, 21 participants died and CHF-related death was defined in 18 patients. Additionally, 106 subjects were hospitalized repetitively due to advance CHF (17 cases in died cohort and 89 cases in survival cohort). Table 1 shows a general characteristic of the patients included in the study. As one can see from Table 1 , no substantial age and gender differences were found among persons who died and survived, as well as differences in body mass index (BMI), glomerular filtration rate (GFR), HbA1c, fasting blood glucose level, blood creatinine level, total cholesterol (TC), low-density lipoprotein cholesterol (LDL-C) and high-density lipoprotein cholesterol (HDL-C), numerous of coronary vessels damaged. No difference was found between the two cohorts in systemic office blood pressure (BP) and heart rate (HR). Documented incidence of type 2 diabetes mellitus in patients of the two cohorts was $38.1 \%$ and $33.8 \%(\mathrm{P}=0.06)$. Note that there was not a statistically significant change in $\AA /$ Àm and $\AA /$ Em between the two cohorts, while decrease in the left ventricular ejection fraction value was quite anticipated in the setting indied patients. At the same time, the level of circulating NT-proBNP was statistically significantly higher in died patients than in survived persons. When analyzing details of pharmacotherapy, no substantial differences were found between the two cohorts with regard to administration of the majority of drugs.

Circulating VE-catherin level in survival and diedpatients Medians of circulating levels of VE-catherin in survived and died patient cohort were $0.63 \mathrm{ng} / \mathrm{ml}$ (95\% confidence interval $[\mathrm{CI}]=0.55-0.64 \mathrm{ng} / \mathrm{ml})$ and $1.03 \mathrm{ng} / \mathrm{ml}(95 \% \mathrm{CI}=0.97-1.07$ $\mathrm{ng} / \mathrm{ml})(\mathrm{P}<0.001)$. The data suggested that VE-catherin plasma levels were directly related to NYHA functional class of $\mathrm{CHF}$ ( $r=0.474, \mathrm{P}=0.006)$, NT-pro-BNP $(\mathrm{r}=0.344, \mathrm{P}=0.001)$, T2DM ( $r=0.42, P=0.006)$, gender $(r=0.366, P<0.001$ for male), multi-vessel lesion of coronary arteries $(\mathrm{r}=0.362, \mathrm{P}=$

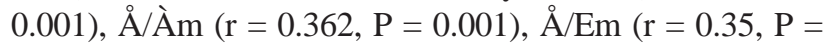
$0.001)$, TC $(r=0.32, P=0.001)$, age $(r=0.28, P=0.001)$, smoking $(\mathrm{r}=0.26, \mathrm{P}=0.001)$ and inversely to LVEF $(\mathrm{r}=-$ $0.426, \mathrm{P}=0.001)$ and eGFR $(\mathrm{r}=-0.416, \mathrm{P}=0.002)$. No significant association between the levels of circulating VEcatherin with fasting plasma glucose, HbA1c, mean systolic and diastolic BP, premature CAD in family anamnesis, and medications for both cohorts of the patients was found.

The predictive value of VE-catherin concentration in study patient population

Table-II

Iterations between sensitivity and specificity of VE-catherin cut-off point level for clinical outcomes in study patient population. Results of the Receive Operation Curve analysis.

\begin{tabular}{lccccc}
\hline & Cut-off point, ng/mL & sensitivity & specificity & AUC (95\% CI) & P-value \\
\hline CHF-related death & 0.755 & 0.990 & 0.550 & $0.953(0.919-0.988)$ & 0.001 \\
CHF-related hospitalization & 0.755 & 0.950 & 0.658 & $0.878(0.819-0.937)$ & 0.001 \\
All-cause mortality & 0.755 & 0.9802 & 0.560 & $0.964(0.936-0.992)$ & 0.001 \\
\hline
\end{tabular}

Note: AUC - area under curve, CI - confidence interval

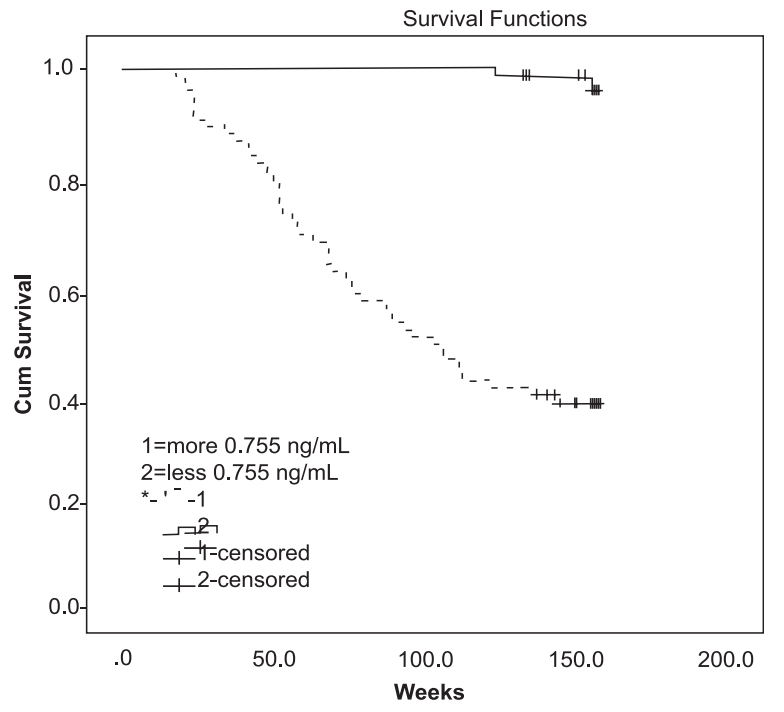

Fig-1: Results of Kaplan-Meier survival analysis: The cumulative survival in two groups patients with low $(<0.755$ $\mathrm{ng} / \mathrm{ml}$ ) and high (>0.755 ng/ml) circulating VE-catherin.
The optimum cut-off point for VE-catherin is determined by the relative importance of the sensitivity and specificity of the test. Receive Operation Curve (ROC) analysis has been shown that cut-off point of VE-catherin concentration for cumulative survival function was $0.755 \mathrm{ng} / \mathrm{ml}$. Area under cure was 0.937 (Std. error $=0.20 ; 95 \% \mathrm{CI}=0.895-0.980$ ), sensitivity and specificity were $96.0 \%$ and $70.6 \%$ respectively. Alterations between sensitivity and specificity of VE-catherin cut-off point level for other clinical outcomes in study patient population are presented in Table 2.For all occasions the model was robust and it has provided a significant results using optimal cut-off point of VE-catherin.

It has been found a significantly divergence of Kaplan-Meier survival curves in patients with high $(>0.755 \mathrm{ng} / \mathrm{ml})$ and low $(<0.755 \mathrm{ng} / \mathrm{ml})$ concentrations of VE-catherin (Figure 1).The curves divergence of events accumulation reached a statistical significance in 26 weeks of observation period $(\mathrm{P}<0.001)$. 
Table-III

Variables Independently Related to 3-years all-cause mortality, CHF-related death, and CHF-related rehospitalisation, obtained by Logistic Regression Analysis.

\begin{tabular}{|c|c|c|c|c|c|c|c|c|c|}
\hline \multirow[t]{2}{*}{ Variables } & \multicolumn{3}{|c|}{ All-cause mortality } & \multicolumn{3}{|c|}{ CHF-related death } & \multicolumn{3}{|c|}{ CHF-related rehospitalisation } \\
\hline & OR & $95 \%$ CI & $\mathrm{P}$ & OR & $95 \%$ CI & $\mathrm{P}$ & OR & $95 \%$ CI & $\mathrm{P}$ \\
\hline VE-catherin & 1.27 & $1.08-1.59$ & 0.002 & 1.16 & $1.02-1.50$ & 0.001 & 1.12 & $1.07-1.25$ & 0.001 \\
\hline NT-pro-BNP & 1.09 & $1.02-1.16$ & 0.002 & 1.42 & $1.22-1.73$ & 0.006 & 1.44 & $1.28-1.67$ & 0.002 \\
\hline LVEF & 1.06 & $1.01-1.12$ & 0.001 & 1.15 & $1.12-1.18$ & 0.014 & 1.22 & $1.07-1.45$ & 0.016 \\
\hline T2DM & 1.05 & $1.01-1.11$ & 0.001 & 1.03 & $0.93-1.10$ & 0.32 & 1.04 & $0.97-1.06$ & 0.42 \\
\hline $\begin{array}{l}\text { three- and multi- } \\
\text { vessel lesion }\end{array}$ & 1.02 & $0.88-1.09$ & 0.56 & 1.01 & $0.92-1.07$ & 0.27 & 1.14 & $1.03-1.26$ & 0.012 \\
\hline
\end{tabular}

Note: OR - odds ratio, CI - confidence interval; LVEF - left ventricular ejection fraction; BNP - brain natriuretic peptide; T2DM - type two diabetes mellitus.

Multivariate logistic regression was used to assess whether any combination of assays was able to better discriminate between survival and died patients. In the logistic regression analysis, the main factors independently related with cumulative mortality and CHF-related rehospitalisations wereVE-catherin, NT-pro-BNP, LVEF, T2DM, and threeand multi-vessel lesion. Circulating VE-catherin independently predicted all-cause mortality $(\mathrm{OR}=1.27 ; 95 \%$ $\mathrm{CI}=1.08-1.59 ; \mathrm{P}=0.002)$, CHF-related death $(\mathrm{OR}=1.16$; 95\% CI 1.02-1.50; P < 0.001), and also CHF-related rehospitalisation $(\mathrm{OR}=1.12 ; 95 \% \mathrm{CI}=1.07-1.25 ; \mathrm{P}<0.001)$ within 3 years of observation period (Table 3).NT-pro-BNP and LVEF remained statistically significant for all categories: all-cause mortality, CHF-related death, and CHF-related rehospitalisations, whereas T2DM and three- and multi-vessel lesionfor all variables did not.

Using a stepwise model selection method for multivariable prediction model we have been investigated the summary effect of any combinations of VE-catherin, NT-pro-BNP, LVEFon all-cause mortality, CHF-related death, and CHFrelated re-hospitalisations. We found that VE-catherin(Model 1) and combination of VE-catherin with NT-pro-BNP (Model 2) remained statistically significant predictors for all-cause mortality (B-coefficient $=1.18, \mathrm{p}=0.001$, and Bcoefficient $=1,13, \mathrm{p}=0.001$ respectively), $\mathrm{CHF}$-related death (B-coefficient=2.36, $\mathrm{p}=0.001$, and B-coefficient $=2,81$, $\mathrm{p}=0.001$ respectively), and CHF-related re-hospitalisations (B-coefficient $=2.34, \mathrm{p}=0.001$, and B-coefficient $=2,26$, $\mathrm{p}=0.001$ respectively), whereas combination of VE-catherin with both NT-pro-BNP and LVEF(Model 3) did not (Bcoefficient $=0.011, p=0.682$, and B-coefficient $=0,012$, $\mathrm{p}=0.588$, and B-coefficient $=0,011, \mathrm{p}=0.568$ respectively). In fact, a stepwise model selection method demonstrated that LVEF, T2DM and three- and multi-vessel lesion of coronary arteries added to combination of VE-catherin and NT-pro-BNPdo not offer any additional information to discriminate between survived and died patients with CHF(B-coefficient of 0.016, 0.086, and 0.018 respectively; p-values of $0.587,0.560$ and 0.540 respectively).

\section{Discussion}

Currently it has been defined that without clinical guidance, echocardiography and natriuretic peptides have suboptimal test characteristics for population-wide CHF risk stratification. ${ }^{13}$ While B-type natriuretic peptide is a strong prognostic indicator in $\mathrm{CHF}$, and possibly superior to traditional prognostic factors, the levels of NT-pro-BNP and BNP must be interpreted according to the clinical picture. ${ }^{14}$ Looking for novel biomarkers are required to be improving reclassification of the risk in CHF over a clinical prediction model. We found that raised level of VE-catherin is associated with a greater risk of death or cardiac events in CHF patients. Because, VE-catherinplays a pivotal role in functional activity ECs, but did not effect on their proliferation and migration, ${ }^{4,5}$ we predispose that VEcatherin might be considered as a marker of endothelial dysfunction. Currently, lack of clinical investigations reflected a prognostic role of VE-catherin in CHF patients. Results of our study suggested that combination of VEcatherinwith traditional prognostic biomarker NT-pro-BNP appears superior to NT-pro-BNP alone and probably has a complementary prognostic value in a patient cohort of CHF. Hens, adding VE-catherin to NT-pro-BNP might be proposed as a suitable tool to improve medical decision making for an identification of CHF patients at high risk. It necessary notes that no any association circulating VE-catherin with demographics parameters, eGFR, fasting glucose, lipids were 
found. Therefore, NYHA class and other cardiovascular risk factors, such as T2DM, produced a strong relation with VEcatherin level, that might be reflected a severity of endothelial dysfunction in evolution of CHF with ischemic origin. It is no clear whether elevated VE-catherin in CHF subjects is a result of ECs damage or opposite an endothelial dysfunction is exerted due to an impact of VE-catherin resulting in other reasons. Further research is necessary to understand the prognostic value of VE-catherin and to identify potential combinations of biological markers that determine the development and prognosis of CHF.

\section{Conclusion:}

Among patients with symptoms of CHF, increased circulating VE-catherin associates with increased 3-year CHF-related death, all-cause mortality, and risk for recurrent hospitalization due to CHF.

\section{Conflict of interests: None.}

\section{Ethical principles}

The study was approved by the local ethics committee of State Medical University, Zaporozhye, Ukraine. The study was carried out in conformity with the Declaration of Helsinki.

\section{Study Restrictions}

This study has some restrictions. The authors believe that a greater cohort of patients with more incidences detected is desirable to improve the power of the study. The authors suppose that these restrictions might have no significant impact on the study data interpretation

\section{Acknowledgement}

We thank all patients for their participation in the investigation, staff of the Regional Zaporozhye Hospital (Ukraine) and the doctors, nurses, and administrative staff in City hospital \# 6 (Zaporozhye, Ukraine), general practices, and site-managed organizations that assisted with the study.

This research received no specific grant from any funding agency in the public, commercial, or not-for-profit sectors.

\section{References}

1. RogerVR. The Heart Failure Epidemic Int J Environ Res Public Health. 2010;7(4):1807-1830.

2. Matsuzawa Y, Sugiyama S, Sumida H, Sugamura K, Nozaki T, Ohba K, Matsubara J, Kurokawa H, Fujisue K, Konishi M, Akiyama E, Suzuki H, Nagayoshi Y, Yamamuro M, Sakamoto K, Iwashita S, Jinnouchi H, Taguri M, Morita S, Matsui K, Kimura K, Umemura S, Ogawa H. Peripheral endothelial function and cardiovascular events in high-risk patients. J Am Heart Assoc. 2013;2(6):426.

3. Young JA, Ting KK, Li J, Moller T, Dunn L, Lu Y, Moses J, Prado-Lourenço L, Khachigian LM, Ng M, Gregory PA, GoodallGJ, Tsykin A, Lichtenstein I, Hahn CN, Tran N,
Shackel N, Kench JG, McCaughan G, Vadas MA, Gamble JR. Regulation of vascular leak and recovery from ischemic injury by general and VE-cadherin-restricted miRNA antagonists of miR-27. Blood. 2013;122(16):2911-9.

4. Giannotta M, Trani M, Dejana E. VE-cadherin and endothelial adherens junctions: active guardians of vascular integrity. Dev Cell. 2013;26(5):441-54.

5. Taha AA, Taha M, Seebach J, Schnittler HJ. ARP2/3mediated junction-associated lamellipodia control VEcadherin-based cell junction dynamics and maintain monolayer integrity. MolBiol Cell 2013 Nov 13.

6. Ley K. Leukocytes talking to VE-cadherin. .Blood. 2013;122(14):2300-1.

7. Suematsu M, Suzuki H, DeLano FA \&Schmid-Schonbein GW The inflammatory aspect of the microcirculation in hypertension:oxidative stress, leukocytes/endothelial interaction, apoptosis. Microcirculation. 2002;9:259-276.

8. McMurray JJV., Adamopoulos S, Anker SD. Auricchio A, Böhm M, Dickstein K. et al. ESC Guidelines for the diagnosis and treatment of acute and chronic heart failure 2012. Eur Heart J. 2012;33:1787-1847.

9. Bluemke DA, Achenbach S, Budoff M, Gerber TC, Gersh B, Hillis LD, et al. Noninvasive coronary artery imaging: magnetic resonance angiography and multidetector computed tomography angiography: a scientific statement from the American Heart Association Committee on Cardiovascular Imaging and Intervention of the Council on Cardiovascular Radiology and Intervention, and the Councils on Clinical Cardiology and Cardiovascular Disease in the Young. Circulation. 2008;118:586-606.

10. Schiller NB., Shah PM., Crawford M, DeMaria A, Devereux $\mathrm{R}$, Feigenbaum $\mathrm{H}$. et al. Recommendations for quantitation of the left ventricle by two-dimensional echocardiography. American Society of Echocardiography Committee on Standards, Subcommittee on Quantitation of TwoDimensional Echocardiograms. J Am SocEchocardiogr. 1989;2:358-367.

11. Pellerin D., Sharma R., Elliott P., Veyrat C. Tissue Doppler, strain, and strain rate echocardiography for the assessment of left and right systolic ventricular function. Heart. 2003;89 (90003):9-17.

12. Levey AS, Stevens LA, Schmid CH, Zhang YL, Castro AF 3rd, Feldman HI. et al. for the CKD-EPI (Chronic Kidney Disease Epidemiology Collaboration). A New Equation to Estimate Glomerular Filtration Rate. Ann Intern Med. 2009;150(9):604-12.

13. Kalogeropoulos AP, GeorgiopoulouVV, deFilippi CR, GottdienerJS, Butler J;Cardiovascular Health Study. Echocardiography, natriuretic peptides, and risk for incident heart failure in older adults: the Cardiovascular Health Study. JACCCardiovasc Imaging. 2012;5(2):131-40.

14. Korenstein D, Wisniversky JP, Wyer P, Adler R, Ponieman $\mathrm{D}, \mathrm{McGinn} \mathrm{T}$. The utility of B-type natriuretic peptide in the diagnosis of heart failure in the emergency department: a systematic review. BMC Emerg. Med. 2007;7:6. 